\title{
The effectiveness of AGV, Ex-PRESS, or trabeculectomy in the treatment of primary and secondary glaucoma: a systematic review and network meta-analysis
}

\author{
Xiwen Zhang ${ }^{1,2,3,4,5} \wedge$, Bo Wang ${ }^{1,2,3,4,5}$, Ruibao Liu ${ }^{1,2,3,4,5}$, Yanchen Chen ${ }^{6} \wedge$, Xiangjie Leng ${ }^{1,2,3,4,5}$, \\ Yukun $\mathrm{Wu}^{1,2,3,4,5}$, Xuejing $\mathrm{Lu}^{1,2,3,5,}$, \\ ${ }^{1}$ Chengdu University of TCM, Chengdu City, China; ${ }^{2}$ Eye College of Chengdu University of TCM, Chengdu, China; ${ }^{3}$ Department of \\ ophthalmology, Ineye Hospital of Chengdu University of TCM, Chengdu, China; ${ }^{4}$ Key Laboratory of Sichuan Province Ophthalmopathy \\ Prevention \& Cure and Visual Function Protection with TCM Laboratory, Chengdu, China; Retinal Image Technology and Chronic Vascular \\ Disease Prevention \& Control and Collaborative Innovation Center, Chengdu University of TCM, Chengdu, China; ${ }^{6} \mathrm{Immunology}$, Ophthalmology \\ and ORL, Faculty of medicine, Complutense University, Madrid, Spain \\ Contributions: (I) Conception and design: All authors; (II) Administrative support: X Lu; (III) Provision of study materials or patients: X Zhang, B \\ Wang, R Liu; (IV) Collection and assembly of data: X Zhang, B Wang, R Liu, Y Chen; (V) Data analysis and interpretation: X Zhang, B Wang, Y \\ Chen, R Liu; (VI) Manuscript writing: All authors; (VII) Final approval of manuscript: All authors. \\ Correspondence to: Xuejing Lu. Chengdu University of TCM, No. 37, Twelfth Bridge Road, Jinniu District, Chengdu 610075, China. \\ Email: luxuejing@cdutcm.edu.cn.
}

Background: In this study, we compared the efficacy of Ahmed, Ex-PRESS, and trabeculectomy to provide a reference for determining surgical schemes for glaucoma patients undergoing external drainage surgery in clinical practice.

Methods: We performed a literature search for studies on the treatment of primary and secondary glaucoma with three types of external drainage surgery (Ahmed, Ex-PRESS, and trabeculectomy). As at April 24, 2021, seven electronic databases were searched for randomized controlled trials comparing any two of Ahmed, Ex-PRESS, and trabeculectomy in the treatment of glaucoma. The Cochrane tool was also adopted to evaluate the risk of bias in these trials. The relative risk (RR) with $95 \%$ confidence interval (CI), and weighted mean difference (WMD) were determined and compared indirectly using $\mathrm{R}$ software.

Results: A total of 14 randomized controlled trials were included in this study, involving 866 eyes of 808 patients. As for the intraocular pressure (IOP) after 3 months, trabeculectomy did not contribute to better improvement than Ahmed (WMD =0.014; 95\% CI: -0.14-0.18) and Ex-PRESS (WMD =0.014; 95\% CI: -0.072-0.097). However, there was a significant difference in the IOP 1 year between trabeculectomy and Ex-PRESS (WMD =0.097; 95\% CI: 0.0080-0.18), with the latter achieving a favorable improvement effect. Meanwhile, the complete success (CS) of trabeculectomy was significantly lower than that of Ex-PRESS (RR $=0.73 ; 95 \%$ CI: $0.57-0.93)$. In addition, Ex-PRESS was superior to Ahmed (WMD $=-0.48 ; 95 \%$ CI: -0.89 to -0.084 ) in terms of a decreased number of post-operative medications.

Discussion: For glaucoma patients who are required to receive external drainage surgery, Ex-PRESS could achieve a significant effect on the IOP 1 year and CS, as well as a marked decrease in the number of postoperative medications used, compared with the other two types of surgery. In terms of the efficacy at least 1 year after surgery, Ex-PRESS should be one of the preferred methods for external drainage.

Keywordsı Effectiveness; trabeculectomy; Ahmed glaucoma valve (AGV); Ex-PRESS

\footnotetext{
^ ORCID: Xiwen Zhang, 0000-0003-4438-8433; Ruibao Liu, 0000-0003-0445-0407; Yanchen Chen, 0000-0002-8544-3323; Xuejing Lu, 0000-0001-5324-4736.
} 
Submitted Dec 09, 2021. Accepted for publication Jan 21, 2022.

doi: 10.21037/apm-21-3968

View this article at: https://dx.doi.org/10.21037/apm-21-3968

\section{Introduction}

Glaucoma is a progressive and irreversible optic nerve injury, which can cause severe progressive visual field defect and even blindness $(1,2)$. Surgical methods are primarily employed for the treatment of advanced or refractory cases and high-risk patients with medication treatment failure (3).

Since the initial introduction of trabeculectomy in 1970, it has been regarded as the gold standard for the surgical treatment of glaucoma, and trabeculectomy-induced complications are well known (4). Trabeculectomy has numerous disadvantages, including several risk factors for younger aged patients, higher preoperative Intraocular Pressure (IOP), diabetes, and postoperative complications (such as significant postoperative inflammation or elevated IOP), which are associated with a higher rate of failure that can limit its success and use (5-7). Recently, miniature glaucoma device implantation (Ex-PRESS) and Ahmed glaucoma valve (AGV) implantation have been considered new surgical alternatives to trabeculectomy $(8,9)$. ExPRESS is a small, stainless steel and splitless glaucoma drainage valve; compared with trabeculectomy, an ExPRESS device has the potential advantage of being less traumatic, as iridectomy and sclerectomy are not required $(10,11)$. AGV has become a recognized effective treatment that significantly improves the success rate of refractory glaucoma surgery (12). AGV implants are equipped with valves, which contribute to the reduced incidence of early low intraocular pressure after surgery and other complications (13).

Recent randomized controlled trials have compared the efficacy and safety of different types of surgeries for the treatment of glaucoma; however, the results are controversial (14-30). This study is the first systematic comparison of three surgical approaches for external drainage, in an attempt to evaluate the differences in the results between Ahmed, Ex-PRESS, and trabeculectomy in the treatment of patients with glaucoma, especially the success rates and complications of the three approaches, with the aim of providing a basis for determining surgical schemes for these patients.

We present the following article in accordance with the Systematic Review Involving a Network Meta-analysis reporting checklist (available at https://apm.amegroups. com/article/view/10.21037/apm-21-3968/rc).

\section{Methods}

\section{Inclusion and exclusion criteria}

\section{Inclusion criteria}

(I) Research type: randomized controlled trials.

(II) Patient group: confirmed diagnosis of glaucoma (all types, including primary and secondary glaucoma); no limitation on the age or gender of patients.

(III) Interventions: the experimental group was treated with glaucoma implants (Ahmed drainage valve or/ and Ex-PRESS drainage device), while the control group was treated with traditional trabeculectomy (excluding modified trabeculectomy); comparative studies involving the Ahmed drainage valve and ExPRESS drainage device.

(IV) Outcome indicators: major indicators including IOP 3 months, IOP 1 year, complete success (CS), overall success (OS), and the number of post-operative medications used; secondary indicators including bestcorrected visual acuity (BCVA) and adverse reactions.

Only articles published in English were eligible for inclusion. Qualified studies were preliminarily included after screening the titles and abstracts, and the full texts of the potential articles were thoroughly reviewed in cases where the eligibility of a study (i.e., whether it met the inclusion criteria) could not be sufficiently determined merely based on its title and abstract.

In the included literature, the preoperative baseline intraocular pressure (IOP baseline) of patients, IOP 3 months, and IOP 1 year (as IOP end-point) was reported (the decreased IOP was represented as a negative value, while the increased IOP was represented as a positive value).

\section{Exclusion criteria}

Non-randomized controlled trials; studies involving patients that were not diagnosed with glaucoma; studies involving the application of other surgical methods besides AGV, Ex-PRESS or trabeculectomy; and studies involving the application of anti-vascular endothelial growth factor (anti- 
VEGF) medications.

\section{Literature retrieval}

Studies on the three types of external drainage surgery (Ahmed, Ex-PRESS, and trabeculectomy) for the treatment of primary and secondary glaucoma published before April 24, 2021 were retrieved from seven electronic databases, including PubMed, Embase, https://www.cochranelibrary. com, Web of Science, ClinicalTrials.gov (http://www. clinicaltrials.gov), Medline (www.medline.com), and Ovid. Subsequently, randomized controlled trials comparing any two of Ahmed, Ex-PRESS, and trabeculectomy were included after screening, and the risk of bias in these trials was evaluated using the Cochrane tool. Furthermore, the $95 \%$ confidence interval (CI), relative risk (RR), and weighted mean difference (WMD) were determined and compared indirectly using $\mathrm{R}$ software (version 4.0.4, GeMTC package). This article is registered in PROSPERO (ID: CRD42021257216).

The methodological quality of each study was assessed by two independent researchers (Zhang $\mathrm{X}$ and Wang B) using the Cochrane collaboration instrument in terms of the following aspects: randomization method, allocation concealment, subject blinding, outcome evaluation blinding, result data integrity, reported outcomes, and other types of bias. Each aspect was assigned a rating of low, high, or uncertain risk based on the results of the quality assessment. Before reaching a consensus, any differences were discussed with the third reviewer (Liu R) for resolution.

\section{Statistical analysis}

The $\mathrm{R}$ software (version 4.0.4, GeMTC package) was adopted to call JAGS 4.3.0 for statistical analysis. The odds ratio $(\mathrm{OR})$ and mean difference $(\mathrm{MD})$ were employed as the effect indicators for counting data and measurement data, respectively. Each effect size was provided with the point estimate and $95 \%$ CI. The random-effects model (REM) of Markov Chain Monte Carlo (MCMC) was adopted and four chains were used for simulation. The number of iterations was set to 50,000, with the first 20,000 iterations for annealing to eliminate the influence of the initial value, and the last 30,000 for sampling. The deviance information criterion (DIC) values of the consistency and inconsistency analyses were compared to evaluate the fitting degree of models. If the difference in the DIC values between both models was $\leq 5$, there would be a consistent fitting degree between both models. Hence, the consistency analysis would be adopted. The node-splitting method was adopted to test the local inconsistency, with $\mathrm{P}<0.05$ indicating the existence of local inconsistency. The "mtc.anohe" command in the "gemtc" package was used to evaluate the global heterogeneity recorded by the variance parameter $\left(\mathrm{I}^{2}\right)$, and values greater than $50 \%$ were considered to be apparent heterogeneity. A funnel plot was employed to evaluate the publication bias. The rank probability plot and relative effect table were employed to rank and describe the effects.

\section{Results}

\section{Data extraction}

In this study, a total of 4,590 relevant articles were retrieved, of which 1,594 duplicates were excluded and another 2,877 were eliminated according to their titles and abstracts, the rest of 119 articles were searched for full-text review, ultimately 17 articles (14-30) were included in review. Due to the fact that one study was repeatedly reported by two articles $(16,25)$ and another was repeatedly reported by three articles $(24,26,30), 14$ clinical randomized controlled trials (RCTs) published from 2003 to 2018 were included in this meta-analysis (14-30). The trial selection process is shown in Figure 1.

\section{Characteristics and quality assessment of the included studies}

The characteristics of the included studies are summarized in Table S1. A total of 14 RCTs (involving 866 eyes from 808 patients) were included in this meta-analysis, including 339 eyes in the trabeculectomy group, 368 eyes in the EX-PRESS group, and 159 eyes in the Ahmed group. Each article was subjected to quality assessment as per the Cochrane Handbook for Systematic Reviews of Interventions (version 5.3). The randomization method was adopted in all 14 studies (14-30) included in this study, among which six studies (14,16,21-26,30) were subjected to the random number table, and the other randomized controlled studies did not provide the specific randomized methods. Allocation concealment was utilized in two studies $(17,23)$, and subject blinding was adopted in one study (17). The data of the 14 studies were complete with no selective reporting bias. The results are summarized in Figures S1,S2 .

\section{Evidence network diagram}

The evidence network diagram of six outcome indicators is 


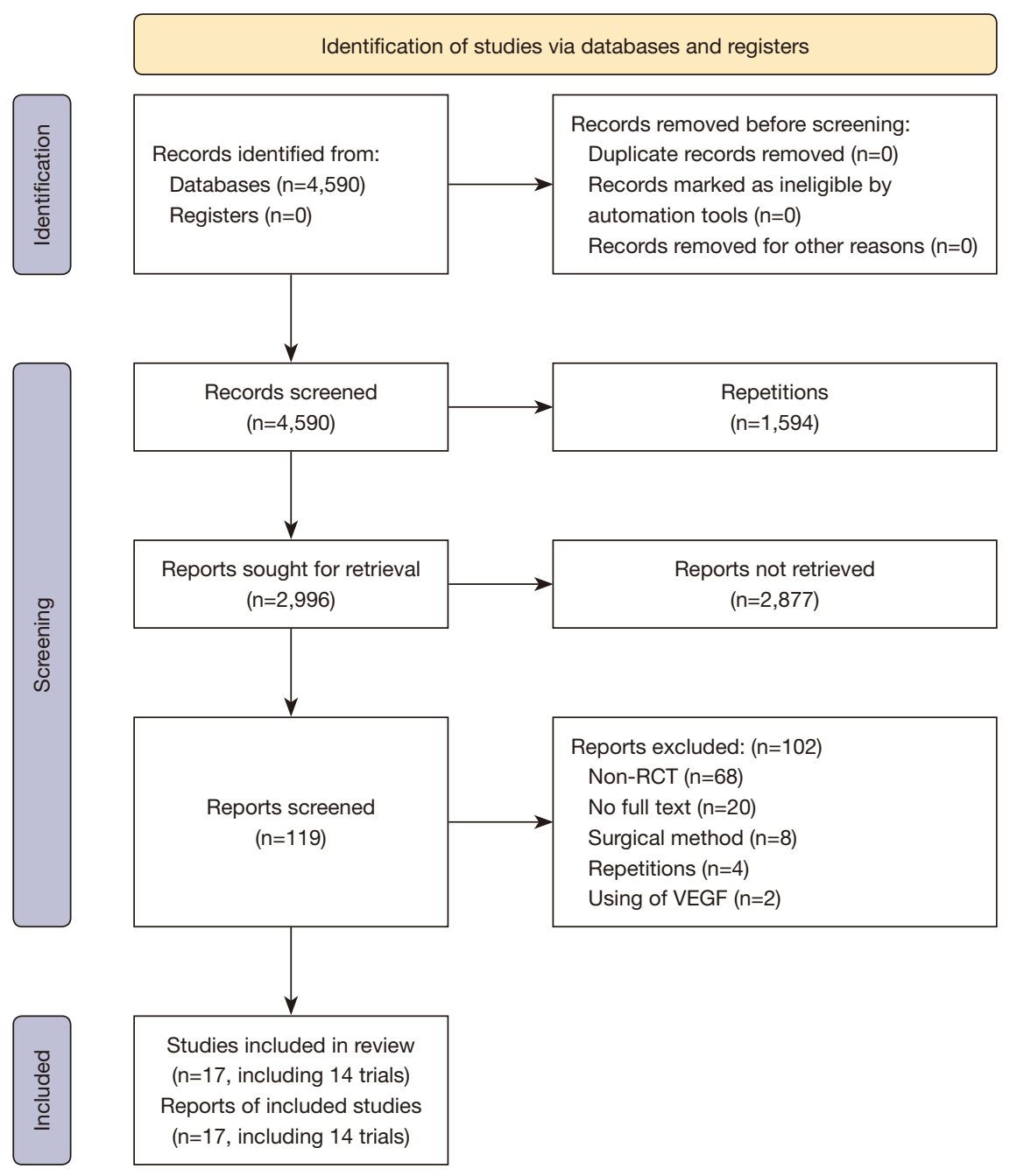

Figure 1 Literature retrieval and selection flowchart.

shown in Figure 2. The thickness of the connecting line is proportional to the number of studies and comparisons.

\section{Network meta-analysis}

\section{Intraocular pressure after 3 months (IOP 3 months)}

Nine of the 14 included studies reported on the IOP 3 months. Summarize from these results, Trabeculectomy had no better improvement than Ahmed (WMD $=0.014$; 95\% CI: $-0.14-0.18$ ) and Ex-PRESS (WMD =0.014; 95\% CI: $-0.072-0.097)$. The ranking results showed that Trabeculectomy had the highest probability of ranking first (0.44), Ex-PRESS had the highest probability of ranking second (0.47) and Ahmed had the highest probability of ranking third (0.44). The pairwise comparison results and detailed ranking results are presented in Table 1, Figure 3 and Table S2. Refer to Figure S3 for the forest plot.

\section{Intraocular pressure after 1 year (IOP 1 year)}

Five of the 14 included studies reported on IOP 1 year. No significant difference in the IOP 1 year was observed between trabeculectomy and Ahmed (WMD $=0.11$; 95\% CI: -0.033-0.25); however, there was a significant difference between trabeculectomy and Ex-PRESS (WMD $=0.097$, 95\% CI: $0.0080-0.18$ ). These results showed that both of these surgical techniques could significantly reduce the IOP, but Ex-PRESS could achieve a better IOP decreased at 1 year than trabeculectomy. Refer to Table 2 for the results of pairwise comparison, Figure S3 for the forest plot, and Figure 3 and Table S2 for detailed ranking results. 


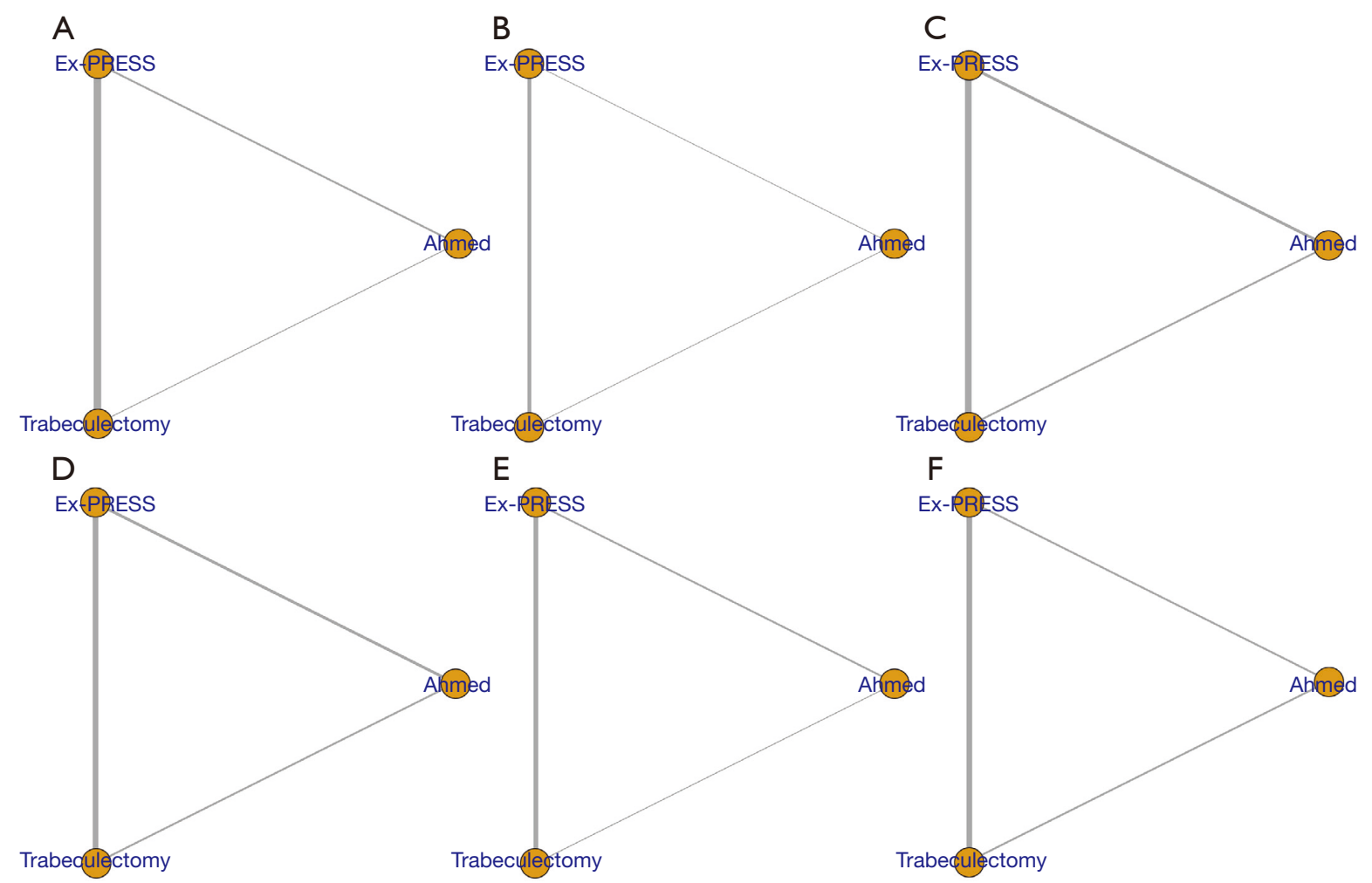

Figure 2 Evidence network diagram of six outcome indicators. (A) IOP 3 months; (B) IOP 1 year; (C) OS; (D) CS; (E) Number of postoperative medications used; (F) BCVA. IOP 3 months, intraocular pressure after 3 months; IOP 1 year, intraocular pressure after 1 year; OS, overall success; CS, complete success; BCVA, best corrected visual acuity.

Table 1 Complications after Ex-PRESS, Ahmed, and trabeculectomy

\begin{tabular}{|c|c|c|c|c|c|}
\hline Name of complications & $\begin{array}{l}\text { Number of } \\
\text { articles }\end{array}$ & \multicolumn{4}{|c|}{ Surgical methods, number of affected eyes/total number of eyes [\%] } \\
\hline Hyphema & 9 & $20 / 97[21]$ & $11 / 254[4.3]$ & $42 / 275[15]$ & $73 / 626[12]$ \\
\hline Choroidal detachment & 7 & $5 / 9[56]$ & $25 / 171[15]$ & 23/163 [14] & $53 / 343[15]$ \\
\hline Shallow anterior chamber & 6 & $11 / 47[23]$ & $28 / 183[15]$ & $25 / 148[17]$ & $64 / 378[17]$ \\
\hline Bleb dysfunction & 1 & $8 / 38[21]$ & $8 / 38[21]$ & / & $16 / 76[21]$ \\
\hline Malignant glaucoma & 1 & $1 / 38[2.6]$ & $0 / 38[0]$ & / & $1 / 76[1.3]$ \\
\hline Implant or tube exposure & 1 & $3 / 59[5]$ & / & $0 / 64[0]$ & $3 / 123[2.4]$ \\
\hline
\end{tabular}

\section{Overall success (OS)}

Ten of the 14 included studies reported on OS. Refer to Table 3 for the results of pairwise comparison, Figure S3 for the forest plot, and Figure 3 and Table S2 for detailed ranking results.

\section{Complete success (CS)}

Nine of the 14 included studies reported on CS. The CS of trabeculectomy was significantly lower than that of ExPRESS (RR $=0.73$; 95\% CI: 0.57-0.93). Refer to Table 3 for the results of pairwise comparison, Figure $\mathrm{S} 3$ for the forest 

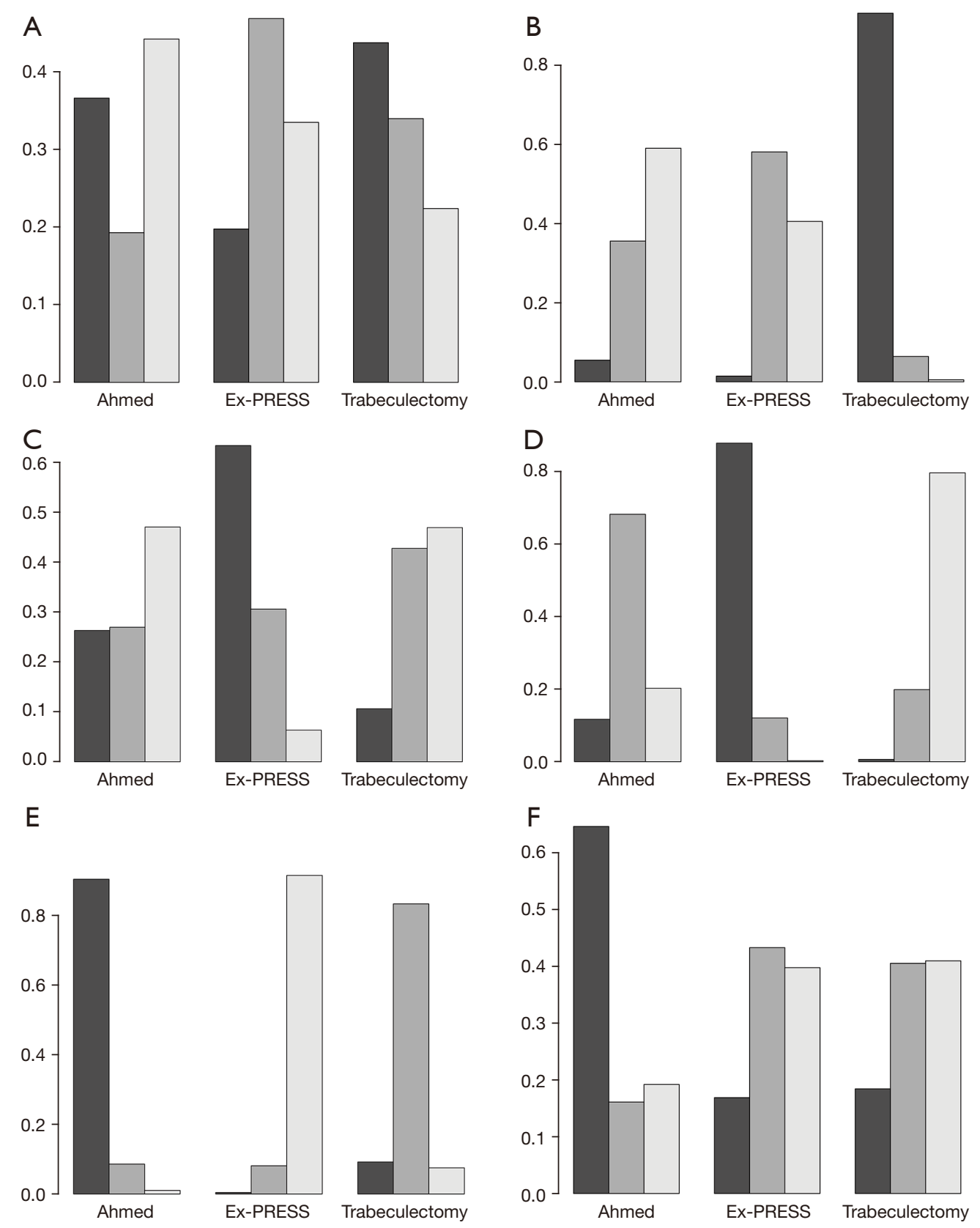

Figure 3 Probability ranking diagram of six outcome indicators in which Black color indicates the No. 1 of ranking; Gray color indicates the No. 2 of ranking; White color indicates the No. 3 of ranking. (A) IOP 3 months; (B) IOP 1 year; (C) OS; (D) CS; (E) Number of postoperative medications used; (F) BCVA. IOP 3 months, intraocular pressure after 3 months; IOP 1 year, intraocular pressure after 1 year; OS, overall success; CS, complete success; BCVA, best corrected visual acuity.

plot, and Figure 3 and Table S2 for detailed ranking results.

\section{The number of post-operative medications used}

Eight of the 14 included studies reporting the number of post-operative medications used. Ex-PRESS was significantly superior (i.e., a decreased number of postoperative medications) to Ahmed (WMD $=-0.48$; 95\% CI: -0.89 to -0.084$)$. Refer to Table 2 for the results of pairwise comparison, Figure S3 for the forest plot, and Figure 3 and Table S2 for detailed ranking results. 
Table 2 Pairwise comparisons analysis results

\begin{tabular}{lccc}
\hline Parameters & Ahmed vs. Ex-PRESS & Ahmed vs. trabeculectomy & Ex-PRESS vs. trabeculectomy \\
\hline BCVA & $0.048(-0.113-0.22)$ & $0.047(-0.118-0.223)$ & $0.00019(-0.1059-0.1031)$ \\
IOP 1 year & $-0.015(-0.159-0.126)$ & $-0.112(-0.251-0.033)$ & $-0.097(-0.178$ to -0.008$)$ \\
IOP 3 months & $-0.001(-0.156-0.136)$ & $-0.015(-0.183-0.138)$ & $-0.013(-0.097-0.073)$ \\
Medicine & $0.48(0.084-0.89)$ & $0.25(-0.162-0.71)$ & $-0.23(-0.537-0.105)$ \\
\hline
\end{tabular}

Data are shown as WMD $(95 \% \mathrm{Cl})$. Differences were statistically significant when $95 \% \mathrm{Cl}$ included 0; differences were not statistically significant and could not be considered significantly different between the two interventions. Comparison in bold refers to the statistically significant comparison. BCVA, best corrected visual acuity; IOP 1 year, intraocular pressure after 1 year; IOP 3 months, intraocular pressure after 3 months; Medicine, number of postoperative medications used; WMD, weighted mean difference ratio; $95 \% \mathrm{Cl}$, $95 \%$ confidence intervals.

Table 3 Pairwise comparisons analysis results

\begin{tabular}{lccc}
\hline Parameters & Ahmed vs. Ex-PRESS & Ahmed vs. trabeculectomy & Ex-PRESS vs. trabeculectomy \\
\hline OS & $0.93(0.72-1.18)$ & $1(0.78-1.29)$ & $1.07(0.93-1.28)$ \\
CS & $0.84(0.6-1.13)$ & $1.15(0.79-1.62)$ & $1.36(1.07-1.77)$ \\
\hline
\end{tabular}

Data are shown as RR $(95 \% \mathrm{Cl})$. Differences were statistically significant when $95 \% \mathrm{Cl}$ includes 1 ; differences were not statistically significant and could not be considered significantly different between the two interventions when RR $>1$, indicating that factor 1 is more effective than factor 2 and less safe than factor 2; when RR $>1$, the opposite. Comparison in bold refers to the statistically significant comparison. OS, overall success; CS, complete success; RR, risk ratio; $95 \% \mathrm{Cl}$ : $95 \%$ confidence intervals.

\section{Best-corrected visual acuity}

Eight of the 14 included studies reported on the BCVA. Refer to Table 2 for the results of pairwise comparison, Figure S3 for the forest plot, and Figure 3 and Table S2 for detailed ranking results.

\section{Analysis of consistency, heterogeneity and publication bias}

The network diagram of these 14 studies formed a closed loop, and there was no inconsistency between the indicators. As for the IOP 3 months, there was significant heterogeneity between the Ex-PRESS and Ahmed groups $\left(\mathrm{I}^{2}=76.8 \%\right.$, $80.1 \%)$, as well as between the trabeculectomy and Ahmed groups $\left(\mathrm{I}^{2}=62.8 \%\right)$, which may have been caused by the differences in the follow-up time, sample size, publication year, and mitomycin-C concentration. As for the decreased number of post-operative medications used, there was significant heterogeneity between the trabeculectomy and Ahmed groups $\left(\mathrm{I}^{2}=64 \%\right)$, but no significant heterogeneity was observed in the other groups. The funnel plot of the 14 included studies was almost symmetrical, which indicated that there was no obvious publication bias. Refer to Figure S4 for heterogeneity analysis and Figure S5 for funnel plot of publication bias analysis.

\section{Safety analysis}

Hyphema, ciliary body detachment, and shallow anterior chamber were the most common postoperative complications. Due to the many adverse reactions and the insufficient number of indicators for each outcome to be used in the meta-analysis, meta-analysis for this aspect was not conducted in this study. The complications after EXPRESS, Ahmed, and trabeculectomy are displayed in Table 1.

\section{Discussion}

Following the 5 th edition of the European Glaucoma Society guidelines (31), the glaucoma can be classified into open-angle glaucoma and angle closure glaucoma to describe the anatomic status of the anterior chamber angle. Each of these is further divided into primary or secondary, indicating the absence or presence, respectively, of other clinically identifiable ocular or systemic disorders to account for the glaucoma.

The first approach in the management of primary openangle glaucoma is usually through topical medications. And then argon laser trabeculoplasty was introduced as a treatment modality for primary open-angle glaucoma by 
Wise and Witter (32).

However, when medication and laser therapy alone is not effective in controlling IOP, more invasive incisional surgery, such as trabeculectomy or glaucoma drainage implants, is indicated.

Although trabeculectomy remains the "gold standard" for glaucoma surgery (4), it is accompanied by high rates of both short- and long-term complications. In the early postoperative period these complications include choroidal effusions, hypotony, shallow anterior chambers, and hyphema. Long-term complications are often bleb related and include leakage, blebitis, and endophthalmitis (5-7).

Currently, minimally invasive glaucoma surgeries (MIGS) have been the latest addition to the glaucoma surgical treatment paradigm (33). Moreover, the latest research points out one of the advantages of the heterogeneous range of available MIGS options is the chance to tailor therapy in an individualized manner (34). Therefore, we try to evaluate the two most common MIGS and the glaucoma surgery "gold standard" for comparison.

In this study, an analysis was performed on 14 highquality clinical randomized controlled studies involving 866 eyes from 808 patients. To the best of our knowledge, this is the first comparative study exploring these three different surgical methods. Through this study, the effectiveness and safety of the three surgical methods were evaluated, and the advantages of Ex-PRESS over the other two were revealed.

Compared with trabeculectomy, the Ex-PRESS implantation group achieved better outcomes in terms of long-term IOP control, CS, and postoperative medication reduction. However, a previous meta-analysis indicated the similar effects of Ex-PRESS implantation and trabeculectomy in reducing IOP (35-38). The reason for this inconsistency may lie in that Ex-PRESS implantation has been developed and gradually perfected as a new technology in recent years, achieving an improved success rate of this surgery with the advancement of clinical research. The most recent literature and opinions were included and analyzed in this study.

The CS of the Ex-PRESS group was better than that of the trabeculectomy group, which is consistent with the findings of previous studies $(13,18,37,39)$, but no significant difference in OS was observed in our study. However, two previous studies $(22,26)$ reported no significant difference in the both the CS and OS between the two groups, which may be attributed to the inconsistent definitions of CS and OS in the existing studies. The difference in standards mainly lies in the postoperative value of
IOP, and is irrelevant to whether medication/surgical intervention is supplemented or not. In addition, in these two studies $(22,26)$, the IOP threshold of OS was defined as $<18 \mathrm{mmHg}$, instead of $<21 \mathrm{mmHg}$. In the other two pairwise comparisons, there was no significant difference in the CS and OS, which is consistent with the findings of previous studies $(14,19,27,28,38)$.

As for the postoperative BCVA, there was no significant difference among the three groups, which is similar to the findings of most studies (14-23).

In this study, the number of postoperative medications used in the Ex-PRESS group was shown to be less than that in the Ahmed group, which is similar to the recent studies by Bo et al. and Zhang et al. $(27,28)$. Meanwhile there was no significant difference between the Ex-PRESS group and the Ahmed group, compared with the trabeculectomy group, which has also been confirmed by multiple studies $(13,16,18,22,24,38,39)$.

The findings of this study revealed that there was no significant difference in the incidence of postoperative complications among the three groups, which is similar to previously reported results $(15,16,18,20,23,29)$. However, the studies of Netland et al. (22) and Dahan et al. (13) indicated that the Ex-PRESS group had a lower overall incidence of postoperative complications than the trabeculectomy group. Ex-PRESS implantation is characterized by less damage to tissues that may be caused by postoperative hyphema, and its incidence was lower in the Ex-PRESS group than that in the other two groups $(36,37,39)$.

Heterogeneity was observed in the current study, which may have been caused by clinical and methodological diversity. In a comparison of 3 -month IOPR\%, the heterogeneity between studies may have been caused by the follow-up time and mitomycin-C concentration. The sources of significant heterogeneity in the OS between studies were not explored due to the inconsistent definitions of the study design levels, quality, and success rate, as well as other factors, such as surgeons' experience, which may contribute to the partial explanation of heterogeneity. Moreover, different participants included in this study suffered from different types of Open Angle Glaucoma (OAG), rather than the simple Primary Open-Angle Glaucoma (POAG), which may be another reason for heterogeneity.

There are also some limitations in this meta-analysis that should be considered. Firstly, publication bias was inevitable, which may also explain the meta-analysis results of Netland 
et al. (22), Arimura et al. (23), and Dahan et al. (17). These studies have all shown "positive results" with registration information provided, which are prone to be accepted and published; meanwhile, similar "negative results" may not have been submitted or published, and some failed studies may have also been included. Secondly, the heterogeneity in this study may have been caused by multiple factors, such as different types of OAG, surgeons' experience, different mitomycin-C concentrations, and different success rate standards. Thirdly, the analysis was not conducted at certain time points as only one trial was involved. For example, the operation success at 4 and 5 years postoperatively were observed by de Jong et al. (16). Also, since Arimura et al. (23) and Dahan et al. (17) only described the IOP control at (9-30) months after surgery, the difference in the long-term efficacy between the three types of surgeries for $>5$ years of follow-up remains unclear. Fourthly, the sensitivity analysis of some outcome indicators was unstable. Therefore, the conclusions were drawn from a thorough interpretation of the summarized results.

\section{Conclusions}

For glaucoma patients who are required to receive external drainage surgery, Ex-PRESS could achieve a significant effect on IOP 1 year and CS, as well as a marked decrease in the number of postoperative medications used, compared with the other two types of surgery. In terms of the efficacy of at least 1 year after surgery, Ex-PRESS should be one of the first choices among external drainage surgeries.

\section{Acknowledgments}

We would like to thank the researchers and study participants for their contributions.

Funding: This work was supported by National Natural Science Foundation of China (82174444), the "Xinglin Scholars" Study on the Protection of Visual Function in TCM (YXRC2020003) and the Youth Innovation Team of Sichuan Traditional Chinese Medicine Industry (2017TD0030).

\section{Footnote}

Reporting Checklist: The authors have completed the Systematic Review Involving a Network Meta-analysis reporting checklist. Available at https://apm.amegroups. com/article/view/10.21037/apm-21-3968/rc
Conflicts of Interest: All authors have completed the ICMJE uniform disclosure form (available at https://apm. amegroups.com/article/view/10.21037/apm-21-3968/coif). All authors report that the financial assistance was provided by the National Natural Science Foundation of China (82174444), the "Xinglin Scholars" Study on the Protection of Visual Function in TCM (YXRC2020003) and the Youth Innovation Team of Sichuan Traditional Chinese Medicine Industry (2017TD0030). The authors have no other conflicts of interest to declare.

Ethical Statement: The authors are accountable for all aspects of the work in ensuring that questions related to the accuracy or integrity of any part of the work are appropriately investigated and resolved.

Open Access Statement: This is an Open Access article distributed in accordance with the Creative Commons Attribution-NonCommercial-NoDerivs 4.0 International License (CC BY-NC-ND 4.0), which permits the noncommercial replication and distribution of the article with the strict proviso that no changes or edits are made and the original work is properly cited (including links to both the formal publication through the relevant DOI and the license). See: https://creativecommons.org/licenses/by-nc-nd/4.0/.

\section{References}

1. Kwon YH, Fingert JH, Kuehn MH, et al. Primary openangle glaucoma. N Engl J Med 2009;360:1113-24.

2. Weinreb RN, Aung T, Medeiros FA. The pathophysiology and treatment of glaucoma: a review. JAMA 2014;311:1901-11.

3. Prum BE Jr, Lim MC, Mansberger SL, et al. Primary OpenAngle Glaucoma Suspect Preferred Practice Pattern(®) Guidelines. Ophthalmology 2016;123:P112-51.

4. Coleman AL. Advances in glaucoma treatment and management: surgery. Invest Ophthalmol Vis Sci 2012;53:2491-4.

5. Rulli E, Biagioli E, Riva I, et al. Efficacy and safety of trabeculectomy vs nonpenetrating surgical procedures: a systematic review and meta-analysis. JAMA Ophthalmol 2013;131:1573-82.

6. Borisuth NS, Phillips B, Krupin T. The risk profile of glaucoma filtration surgery. Curr Opin Ophthalmol 1999;10:112-6.

7. Nouri-Mahdavi K, Brigatti L, Weitzman M, et al. Outcomes of trabeculectomy for primary open-angle 
glaucoma. Ophthalmology 1995;102:1760-9.

8. Gandham SB, Costa VP, Katz LJ, et al. Aqueous tubeshunt implantation and pars plana vitrectomy in eyes with refractory glaucoma. Am J Ophthalmol 1993;116:189-95.

9. Hille K, Moustafa B, Hille A, et al. Drainage devices in glaucoma surgery. Klin Oczna 2004;106:670-81.

10. Hill R, Ohanesian R, Voskanyan L, et al. The Armenian Eye Care Project: surgical outcomes of complicated paediatric glaucoma. Br J Ophthalmol 2003;87:673-6.

11. Stewart RM, Diamond JG, Ashmore ED, et al. Complications following ex-press glaucoma shunt implantation. Am J Ophthalmol 2005;140:340-1.

12. Tavolato M, Babighian S, Galan A. Spontaneous extrusion of a stainless steel glaucoma drainage implant (Ex-PRESS). Eur J Ophthalmol 2006;16:753-5.

13. Dahan E, Carmichael TR. Implantation of a miniature glaucoma device under a scleral flap. J Glaucoma 2005;14:98-102.

14. Wilson MR, Mendis U, Paliwal A, et al. Long-term follow-up of primary glaucoma surgery with Ahmed glaucoma valve implant versus trabeculectomy. Am J Ophthalmol 2003;136:464-70.

15. Mendoza-Mendieta ME, López-Venegas AP, Valdés-Casas G. Comparison between the EX-PRESS P-50 implant and trabeculectomy in patients with open-angle glaucoma. Clin Ophthalmol 2016;10:269-76.

16. de Jong L, Lafuma A, Aguadé AS, et al. Five-year extension of a clinical trial comparing the EX-PRESS glaucoma filtration device and trabeculectomy in primary open-angle glaucoma. Clin Ophthalmol 2011;5:527-33.

17. Dahan E, Ben Simon GJ, Lafuma A. Comparison of trabeculectomy and Ex-PRESS implantation in fellow eyes of the same patient: a prospective, randomised study. Eye (Lond) 2012;26:703-10.

18. Errico D, Scrimieri FL, Riccardi R, et al. Trabeculectomy Versus Ex-Press Glaucoma Filtration Device in Silicomacrophagocytic Open Angle Glaucoma Secondary to Silicone Oil Emulsification. Middle East Afr J Ophthalmol 2016;23:177-82.

19. Pakravan M, Homayoon N, Shahin Y, et al. Trabeculectomy with mitomycin $\mathrm{C}$ versus Ahmed glaucoma implant with mitomycin $\mathrm{C}$ for treatment of pediatric aphakic glaucoma. J Glaucoma 2007;16:631-6.

20. Kobayashi N, Hirooka K, Nitta E, et al. Visual acuity and corneal higher-order aberrations after EX-PRESS or trabeculectomy, and the determination of associated factors that influence visual function. Int Ophthalmol 2018;38:1969-76.
21. El-Saied HM, Abdelhakim MASE. Different surgical modalities for management of persistent glaucoma after silicone oil removal in vitrectomized eyes: One Year Comparative Study. Retina 2017;37:1535-43.

22. Netland PA, Sarkisian SR Jr, Moster MR, et al. Randomized, prospective, comparative trial of EX-PRESS glaucoma filtration device versus trabeculectomy (XVT study). Am J Ophthalmol 2014;157:433-440.e3.

23. Arimura S, Miyake S, Iwasaki K, et al. Randomised Clinical Trial for Postoperative Complications after ExPRESS Implantation versus Trabeculectomy with 2-Year Follow-Up. Sci Rep 2018;8:16168.

24. Wagschal LD, Trope GE, Jinapriya D, et al. Prospective Randomized Study Comparing ExPRESS to Trabeculectomy: 1-Year Results. J Glaucoma 2015;24:624-9.

25. de Jong LA. The Ex-PRESS glaucoma shunt versus trabeculectomy in open-angle glaucoma: a prospective randomized study. Adv Ther 2009;26:336-45.

26. Gonzalez-Rodriguez JM, Trope GE, Drori-Wagschal L, et al. Comparison of trabeculectomy versus Ex-PRESS: 3-year follow-up. Br J Ophthalmol 2016;100:1269-73.

27. Bo W, Dai D, Sun F. Observation of curative effects of Ex-PRESS and AGV implantation in the treatment of refractory glaucoma. Exp Ther Med 2018;15:4419-25.

28. Zhang M, Li B, Sun Y. EX-PRESS and ahmed glaucoma valve in treatment of refractory glaucoma. Acta Ophthalmol 2016;94:e382-3.

29. Kato N, Takahashi G, Kumegawa K, et al. Indications and postoperative treatment for Ex-PRESS(®) insertion in Japanese patients with glaucoma: comparison with standard trabeculectomy. Clin Ophthalmol 2015;9:1491-8.

30. Beltran-Agullo L, Trope GE, Jin Y, et al. Comparison of visual recovery following ex-PRESS versus trabeculectomy: results of a prospective randomized controlled trial. J Glaucoma 2015;24:181-6.

31. European Glaucoma Society Terminology and Guidelines for Glaucoma, 5th Edition. Br J Ophthalmol 2021;105:1-169.

32. Wise JB, Witter SL. Argon laser therapy for open-angle glaucoma. A pilot study. Arch Ophthalmol 1979;97:319-22.

33. Mathew DJ, Buys YM. Minimally Invasive Glaucoma Surgery: A Critical Appraisal of the Literature. Annu Rev Vis Sci 2020;6:47-89.

34. Gillmann K, Mansouri K. Minimally Invasive Glaucoma Surgery: Where Is the Evidence? Asia Pac J Ophthalmol (Phila) 2020;9:203-14.

35. Wang W, Zhang X. Meta-analysis of randomized controlled trials comparing EX-PRESS implantation 
with trabeculectomy for open-angle glaucoma. PLoS One 2014;9:e100578.

36. Chen G, Li W, Jiang F, et al. Ex-PRESS implantation versus trabeculectomy in open-angle glaucoma: a metaanalysis of randomized controlled clinical trials. PLoS One 2014;9:e86045.

37. Wang $W$, Zhou $M$, Huang $W$, et al. Ex-PRESS implantation versus trabeculectomy in uncontrolled glaucoma: a meta-analysis. PLoS One 2013;8:e63591.

38. HaiBo T, Xin K, ShiHeng L, et al. Comparison of Ahmed

Cite this article as: Zhang X, Wang B, Liu R, Chen Y, Leng X, Wu Y, Lu X. The effectiveness of AGV, Ex-PRESS, or trabeculectomy in the treatment of primary and secondary glaucoma: a systematic review and network meta-analysis. Ann Palliat Med 2022;11(1):321-331. doi: 10.21037/apm-21-3968 glaucoma valve implantation and trabeculectomy for glaucoma: a systematic review and meta-analysis. PLoS One 2015;10:e0118142.

39. Sun Y, Zhang B, Zhou R, et al. Trabeculectomy and EXPRESS Implantation in Open-Angle Glaucoma: An Updated Meta-Analysis of Randomized Controlled Trials. J Ophthalmol 2019;2019:2071506.

(English Language Editor: A. Kassem) 


\section{Supplementary}

Table S1 Characteristics included in all studies

\begin{tabular}{|c|c|c|c|c|c|c|c|}
\hline No. & Author & Country & Design & EX/A/TR & Glaucoma types & Age, years & Follow-up time \\
\hline 2 & $\begin{array}{l}\text { de Jong et al. 2011, (16); de Jong } \\
\text { 2009, (25) }\end{array}$ & Netherlands & $\mathrm{RCT}$ & 40/NA/39 & POAG, PXFG, PG & $65.6 \pm 13.4$ & 5 years \\
\hline 3 & Errico et al. 2016, (18) & Italy & $\mathrm{RCT}$ & 10/NA/16 & OAG & $53.9 \pm 13.9$ & $\geq 2$ years \\
\hline 6 & Netland et al. 2014, (22) & American & RCT & $59 / N A / 61$ & OAG (including POAG, and PG); & $68.6 \pm 11.0$ & 2 years \\
\hline 7 & Arimura et al. 2018, (23) & Japan & $\mathrm{RCT}$ & $32 / N A / 32$ & POAG, PXFG & $71.7 \pm 10.5$ & 3 months \\
\hline 8 & Mendoza-Mendieta et al. 2016, (15) & Mexico & RCT & 20/NA/19 & POAG, PXFG PG; secondary OAG & $64.3 \pm 14.6$ & $9.1 \pm 5.1$ months \\
\hline 12 & El-Saied et al. 2017, (21) & Egypt & $\mathrm{RCT}$ & $10 / 10 / 10$ & Secondary OAG & $34.7 \pm 4.9$ & 1 years \\
\hline 13 & $\begin{array}{l}\text { Wagschal et al. 2015, (24); } \\
\text { Gonzalez-Rodriguez et al. 2016, } \\
\text { (26); Beltran-Agullo et al. 2015, (30) }\end{array}$ & Canada & RCT & 33/NA/31 & OAG & $63.9 \pm 11.6$ & 3 years \\
\hline 14 & Dahan et al. 2012, (17) & South Africa & RCT & 15/NA/15 & Bilateral POAG & $65.4 \pm 13.7$ & $23.6 \pm 6.9$ months \\
\hline
\end{tabular}

$\mathrm{RCT}$, randomized controlled trials; NVG, neovascular glaucoma; OAG, open angle glaucoma; POAG, primary open-angle glaucoma; PXFG, pseudo exfoliation glaucoma; PG: pigmentary glaucoma; PCACG, primary chronic angle-closure glaucoma.

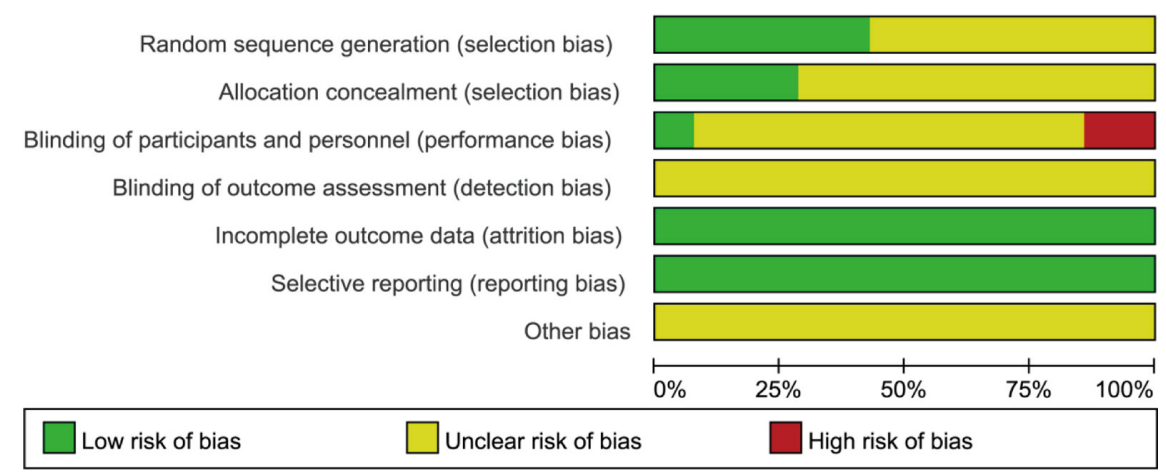

Figure S1 Judging the risk of bias for each project as a percentage of all eligible. 


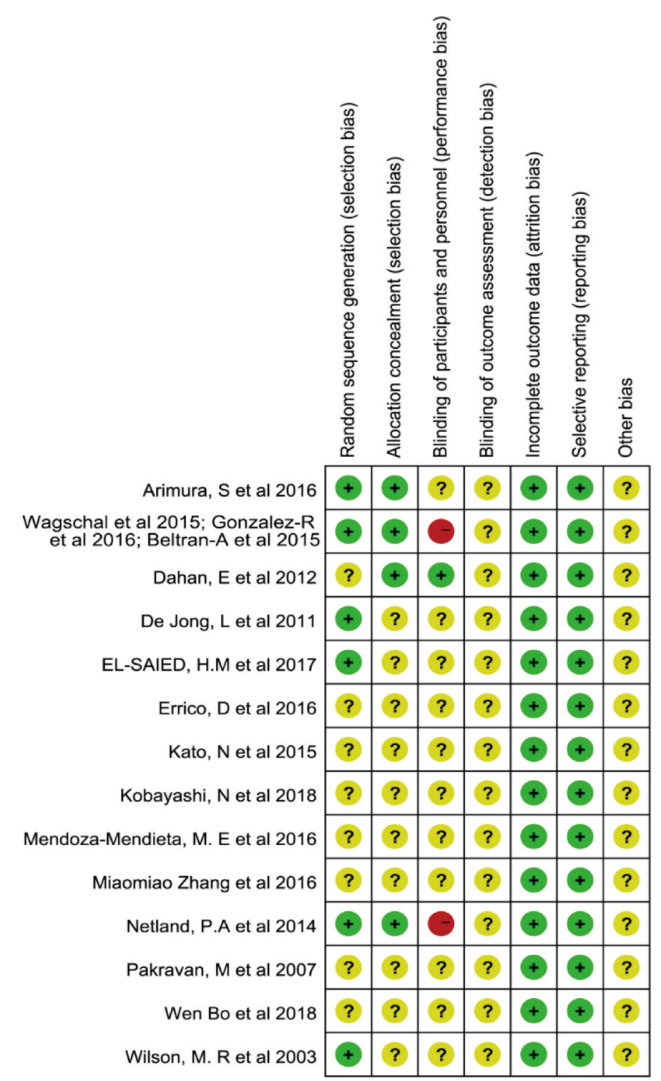

Figure S2 Judgment of the risk of bias for each study.

Table S2 Probability ranking table of 6 outcome indicators

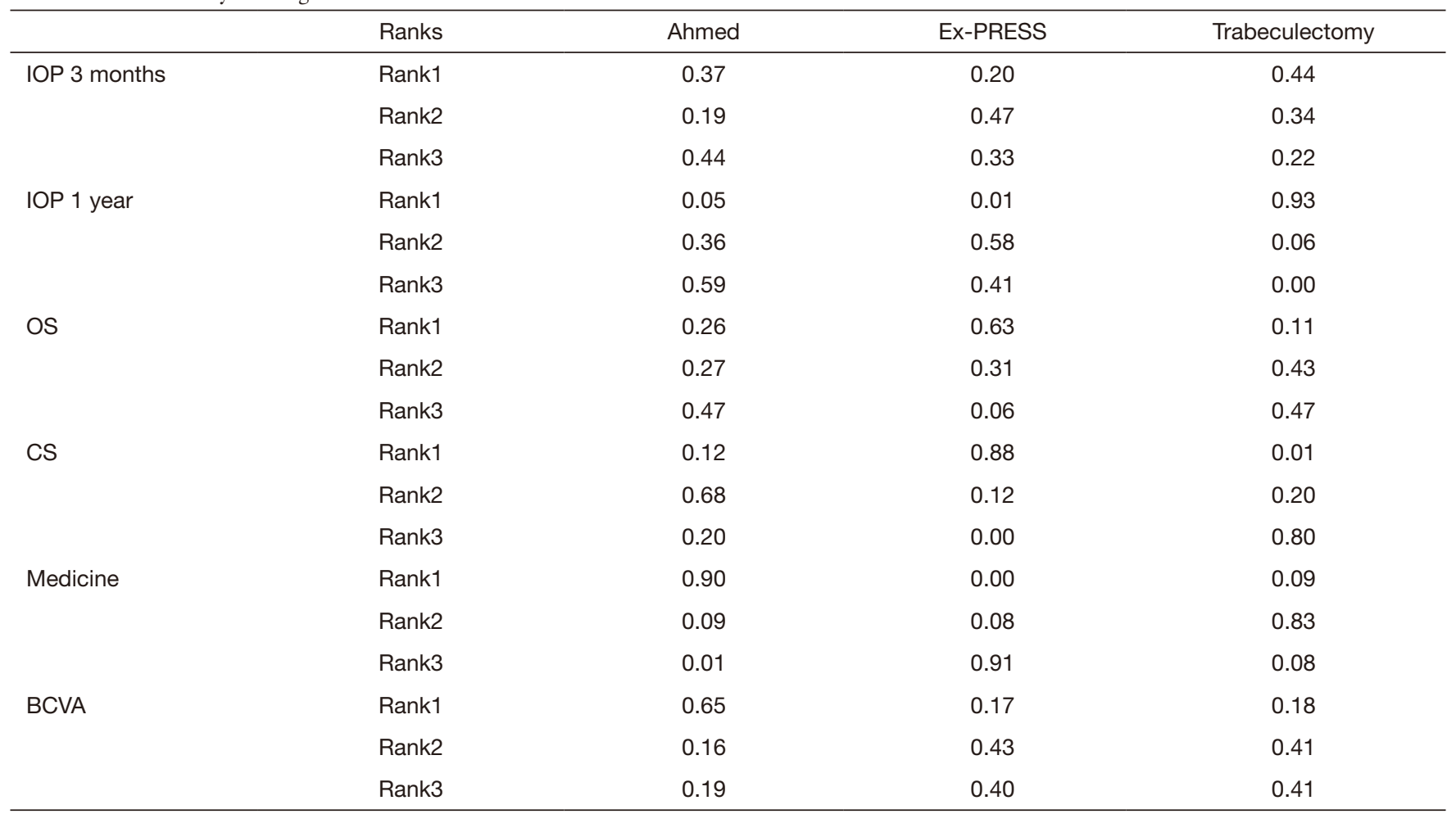



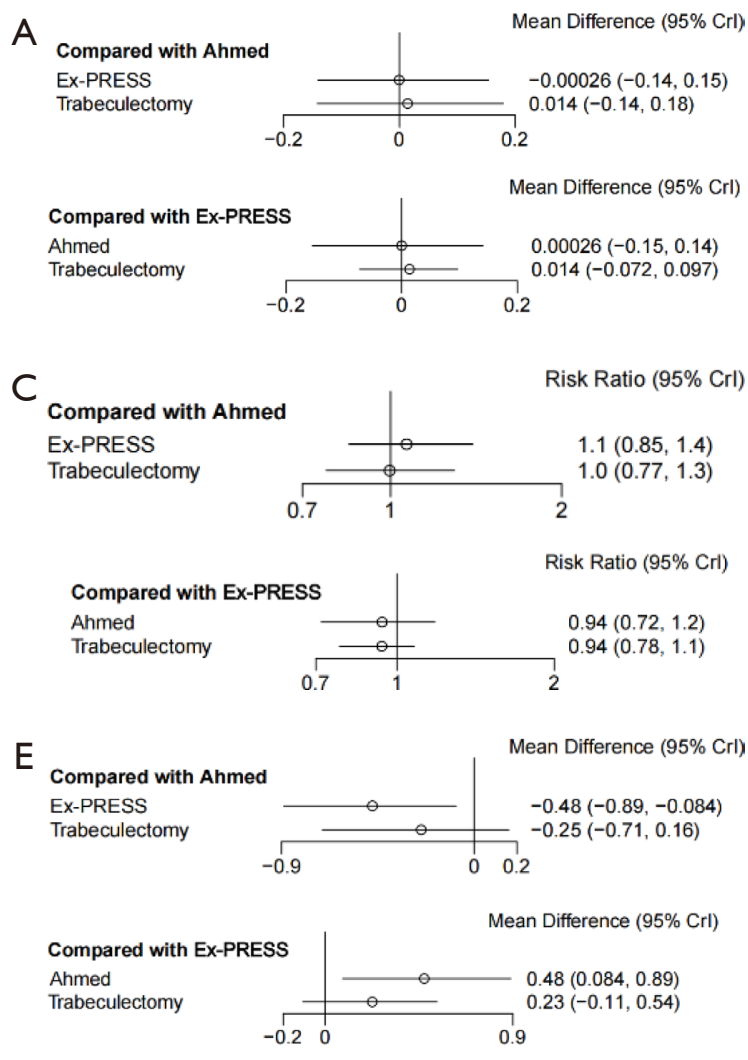

B
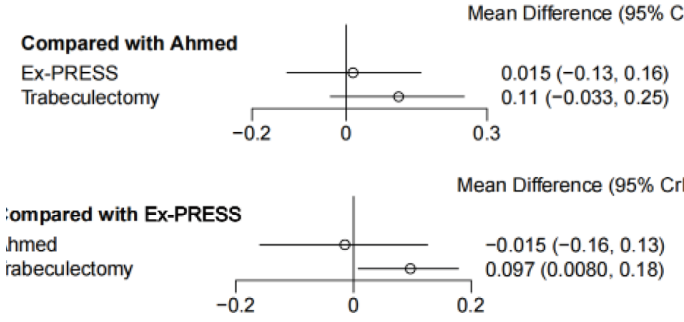

D

Compared with Ahmed Ex-PRESS

Trabeculectomy

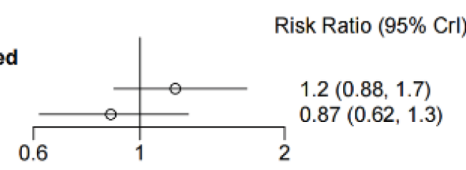

2

Risk Ratio $(95 \% \mathrm{Crl})$

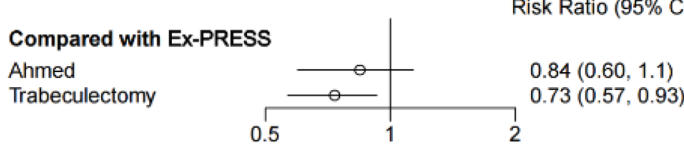

F Compared with Ahmed EX-PRESS

Trabeculectomy

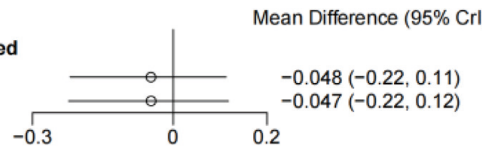

Mean Difference $(95 \% \mathrm{Crl})$

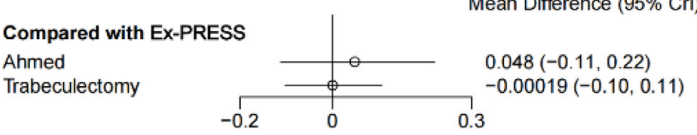

Figure S3 Forest plot of of six outcome indicators. (A) Intraocular pressure after 3 months (IOP 3 months); (B) intraocular pressure after 1 year (IOP 1 year); (C) overall success (OS); (D) complete success (CS); (E) the number of post-operative medications used; (F) best corrected visual acuity (BCVA). 
A

Study
Ex-PRESS vs Ahmed
HEBA MAGDY EL-SAIED et al
Wen Bo et al
Pooled (pair-wise)
Indirect (back-calculated)
Pooled (network)
Trabeculectomy vs Ahmed
HEBA MAGDY EL-SAIED et al
Pooled (pair-wise)
Indirect (back-calculated)
Pooled (network)
Trabeculectomy vs Ex-PRESS
Beltran-Agullo, Laura et al
Donato Errico et al
HEBA MAGDY EL-SAIED et al
Leo de Jong et al
Mendoza-Mendieta, M. E et al
Nobuko Kobayashi et al
Noriko Kato et al
Shogo Arimura et al
Pooled (pair-wise)
Indirect (back-calculated)
Pooled (network)

$76.8 \%$

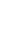

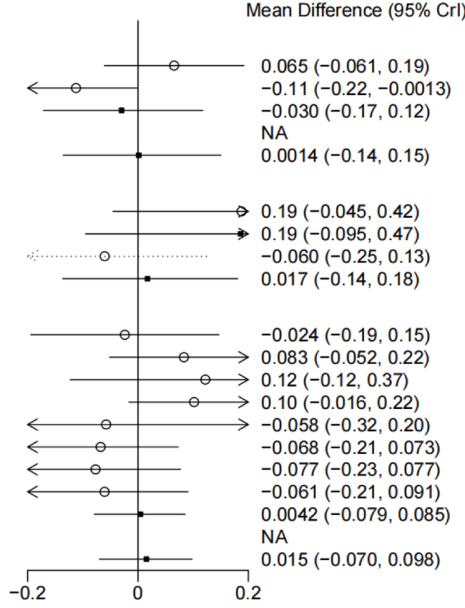

B

Study

Ex-PRESS vs Ahmed

HEBA MAGDY EL-SAIED et al

Pooled (pair-wise)

Indirect (back-calculated)

Pooled (network)

Trabeculectomy vs Ahmed

HEBA MAGDY EL-SAIED et al

Pooled (pair-wise)

Indirect (back-calculated)

Pooled (network)

Trabeculectomy vs Ex-PRESS

Beltran-Agullo, Laura et al

Donato Errico et al

HEBA MAGDY EL-SAIED et al

Leo de Jong et al

Mendoza-Mendieta, M. E et al

Pooled (pair-wise)

Pooled (network)

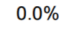

$0.0 \%$

$\wedge^{\wedge} 2$

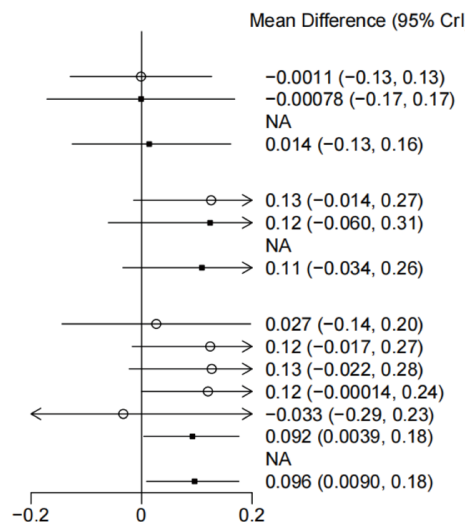

D

Study ${ }^{\wedge} 2$

Ex-PRESS vs Ahmed

HEBA MAGDY EL-SAIED et al

Miaomiao Zhang et al

Wen Bo et al

Pooled (pair-wise)

Indirect (back-calculated)

Pooled (network)

Trabeculectomy vs Ahmed

HEBA MAGDY EL-SAIED et a

Mohammad Pakravan et al

Pooled (pair-wise)

Indirect (back-calculated)

Pooled (network)

Trabeculectomy vs Ex-PRESS

Beltran-Agullo, Laura et al

Donato Errico et al

HEBA MAGDY EL-SAIED et al

Leo de Jong et al
Mendoza-Mendieta, M. E et al

Noriko Kato et al

Noriko Kato et al

Indirect (back-calculated)

Pooled (network)

$0.0 \%$

$1.5(0.88,2.5)$

$0.99(0.86,1.2)$

$14.4 \%$

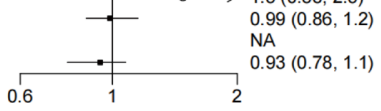

$\wedge 2$

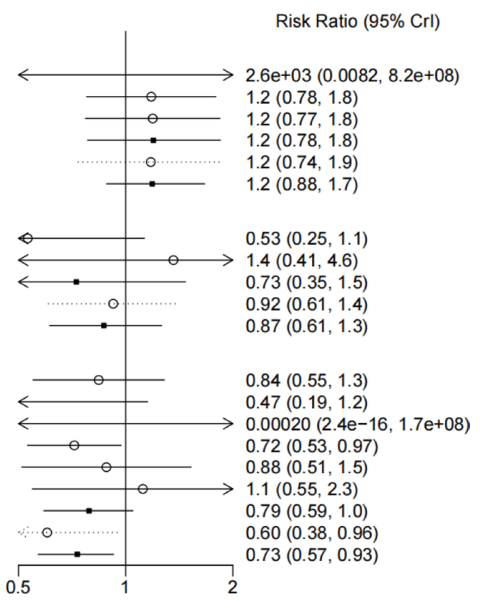

$\mathrm{F}$

E

Study ${ }^{\wedge 2}$

Ex-PRESS vs Ahmed

Miaomiao Zhang et al

Wen Bo et al

Pooled (pair-wise) $\quad 0.0 \%$

$\begin{array}{ll}\text { Indirect (back-calculated) } & \\ \text { Pooled (network) } & 19.5 \%\end{array}$

Trabeculectomy vs Ahmed

M. ROY WILSON et al

Pooled (pair-wise)

Indirect (back-calculated)

Pooled (network)

$64.0 \%$

Trabeculectomy vs Ex-PRESS

Beltran-Agullo, Laura et al

Dahan, E. et al

Donato Errico et al

Leo de Jong et al

Netland, Peter $A$ et a

Pooled (pair-wise)

Indirect (back-calculated)

Pooled (network)

$26.4 \%$

. $\%$

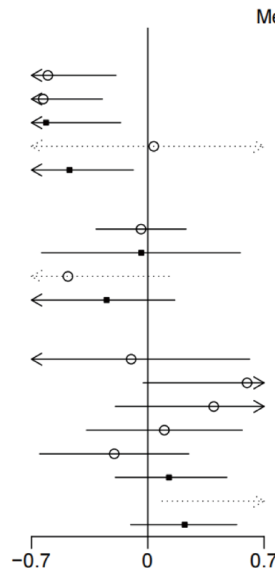

Mean Difference $(95 \%$ Crl) Study

Ex-PRESS vs Ahmed

HEBA MAGDY EL-SAIED et al

Wen Bo et al

Pooled (pair-wise)

Indirect (back-calculated)

Pooled (network)

Trabeculectomy vs Ahmed

HEBA MAGDY EL-SAIED et al

Mohammad Pakravan et al

Pooled (pair-wise)

Indirect (back-calculated)

Pooled (network)

Trabeculectomy vs Ex-PRESS

Beltran-Agullo, Laura et al

HEBA MAGDY EL-SAIED et

Mendoza-Mendieta, M. E et al

Netland, Peter A et al

Nobuko Kobayashi et al

Shogo Arimura

Pooled (pair-wise)

Indirect (back-calculated)

$33.9 \%$

$\wedge^{\wedge} 2$

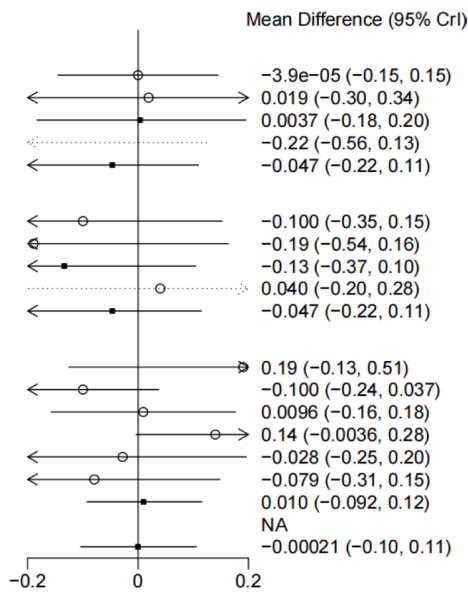

Figure S4 Heterogeneity Analysis of of six outcome indicators. (A) Intraocular pressure after 3 months (IOP 3 months); (B) intraocular pressure after 1 year (IOP 1 year); (C) overall success (OS); (D) complete success (CS); (E) the number of post-operative medications used; (F) best corrected visual acuity (BCVA). 

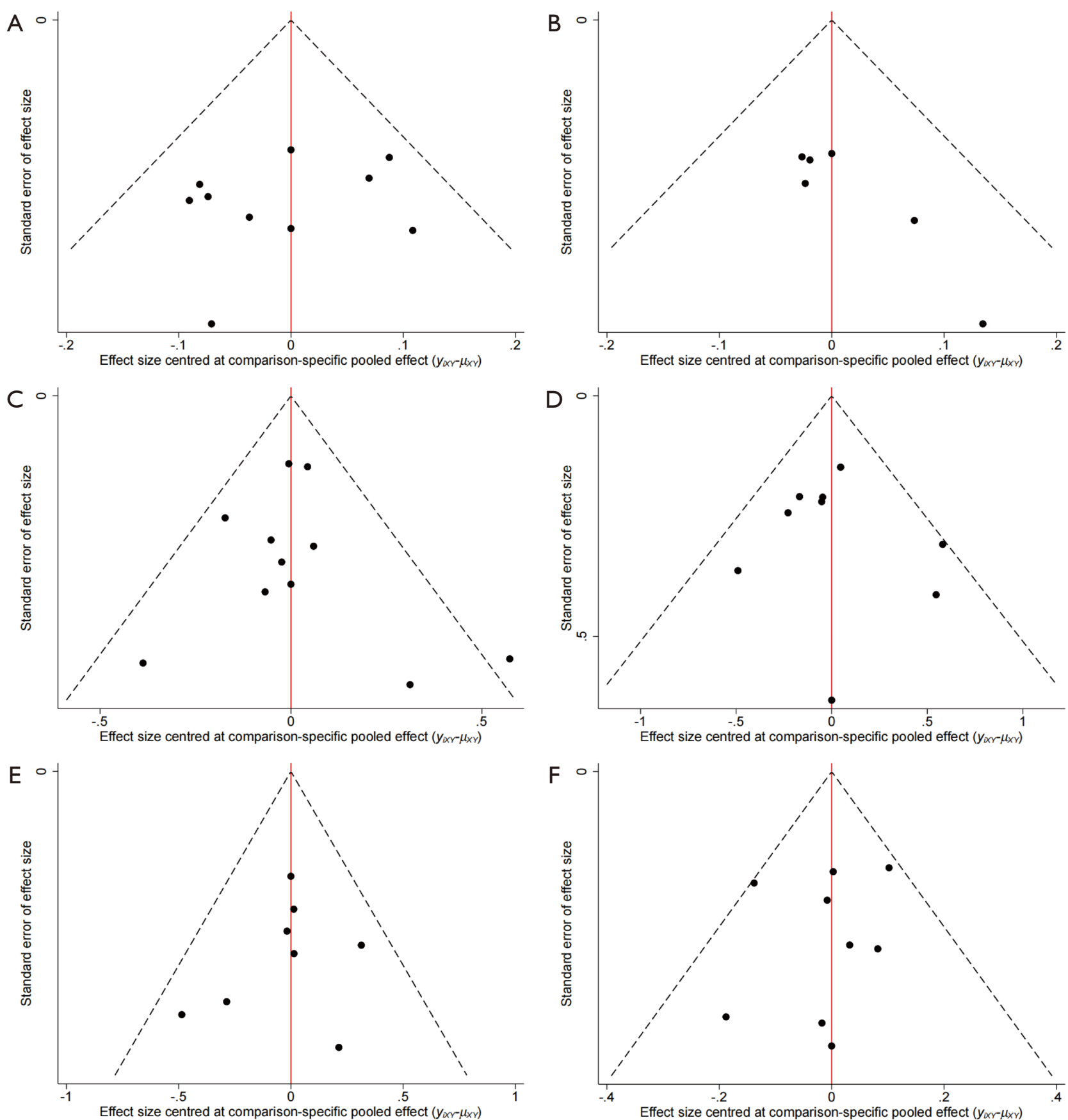

Figure S5 Funnel plot of of six outcome indicators. (A) Intraocular pressure after 3 months (IOP 3 months); (B) intraocular pressure after 1 year (IOP 1 year); (C) overall success (OS); (D) complete success (CS); (E) the number of post-operative medications used; (F) best corrected visual acuity (BCVA). 